\title{
In vivo intravital endoscopic confocal fluorescence microscopy of normal and acutely injured rat lungs
}

\author{
Frederic Chagnon ${ }^{1,6}$, Clement Fournier ${ }^{1,2,6}$, Paul G Charette ${ }^{2}$, Luc Moleski ${ }^{1,3}$, Marcel D Payet ${ }^{4}$, Leland G Dobbs ${ }^{5}$ and \\ Olivier Lesur ${ }^{1,3}$
}

We present a new lung imaging technique based on endoscopic confocal fluorescence microscopy (ECFM), which is a new method that is able to provide cellular and structural assessment of living tissue using a small confocal probe in direct contact with the visceral pleura. To observe distal airspace structure and cellular condition in normal and injured lungs (hyperoxic and bleomycin challenged), we used fluorescent-specific marker contrast and ECFM. Alveolar space ECFM with spectral analyses were performed at 488-nm excitation using FITC-labeled markers or naturally fluorescent dyes. The normal lung was compared with the sick lung, where our in vivo imaging experiments correlated well with results obtained with corresponding ex vivo conventional assays. Four main elements pertaining to the acute lung injury/ acute respiratory distress syndrome (ALI/ARDS) pathophysiology and established early key events were specifically studied: alveolar epithelial membrane phenotype, lung cell apoptosis, neutrophil recruitment, and edema. ECFM allowed visualization of (i) fine-tuned ultrastructural lectin (RCA-1) and sialoglycoprotein (RTI40) epithelial cell membrane expression, (ii) YO-PRO-1-related DNA linking of lung cell apoptosis, (iii) PKH2 green fluorescent cell linker-labeled neutrophil tracking in lung microcirculatory network and airspaces, (iv) FITC-dextran plasma contrast and extravasation with edema formation. ECFM provides reliable results to corresponding ex vivo fluorescent methods. ECFM, using the minimally invasive Five-1 $\mathbb{R}$ optical instrument and specific fluorescent markers, is able to provide real-time potentially useful imaging of live unfixed normal and injured lung tissue with promising developments for improving bedside diagnostic and decision-making therapeutic strategy in patients with ALI.

Laboratory Investigation (2010) 90, 824-834; doi:10.1038/labinvest.2010.76; published online 12 April 2010

KEYWORDS: lung microimaging in vivo; ALI/ARDS; epithelium; apoptosis; edema

Acute lung injury (ALI) and acute respiratory distress syndrome (ARDS) are critical conditions characterized by a severe acute hypoxemic respiratory failure and bilateral lung infiltrates not related to left-sided heart failure. ${ }^{1}$ The incidence of ALI/ARDS averages 80 per 100000 person-years in developed countries, ${ }^{2,3}$ with significant morbidity and a 'reallife and real-time' mortality rate of $30-40 \% .^{4,5}$

ALI/ARDS must be differentiated from other acute pulmonary disease entities but there are no 'easy-to-do' diagnostic tests. Available tools include (i) chest imaging and bronchoscopy with bronchoalveolar lavage, which are often not discriminant when pneumonia is not causative, and (ii) open lung biopsy, which is a gold standard for accurate diagnosis, but with relatively low benefit/harm ratio in some places. ${ }^{6,7}$ Critical care physicians must otherwise rely on the widely accepted criteria of diffuse infiltrates on chest imaging and severe hypoxemia, and on the presence of known risk factors. ${ }^{1,8}$

Histologically, ALI/ARDS is defined by a diffuse alveolar damage (DAD), which is at early stage formed into edema, necrosis of type I pneumocytes and endothelial cells, sparse interstitial inflammatory infiltrate, intra-alveolar hemorrhage, fibrin deposition, and later on type II pneumocyte proliferation, with sometimes hyaline membranes and

\footnotetext{
${ }^{1}$ Groupe de Recherche en Physiopathologie Pulmonaire, Centre de Recherche Clinique, CHUS, Sherbrooke, QC, Canada; ${ }^{2}$ Department of Electrical and Computer Engineering, Faculty of Engineering, University of Sherbrooke, Sherbrooke, QC, Canada; ${ }^{3}$ Medical Intensive Care Unit, Department of Medicine, Faculty of Medicine, University of Sherbrooke, Sherbrooke, QC, Canada; ${ }^{4}$ Department of Physiology and Biophysics, University of Sherbrooke, QC, Canada and ${ }^{5}$ Cardiovascular Research Institute, University of California, San Francisco, CA, USA

Correspondence: Dr O Lesur, MD, PhD, Department of Medicine, CHUS, FMSSS, University of Sherbrooke, 3001, 12th Avenue Nord, Sherbrooke, Quebec, Canada J1H $5 \mathrm{~N} 4$.

E-mail: Olivier.Lesur@USherbrooke.ca

${ }^{6}$ These authors contributed equally to this paper.

Received 1 August 2009; revised 14 February 2010; accepted 22 February 2010
} 
fibrosis, when the repair is failing. ${ }^{9}$ The earliest phases of $\mathrm{DAD}$, the ones that should be targeted for therapeutic interventions, is exudative and characterized by neutrophilic infiltrate and edema, and alveolar epithelial cell repair is a cornerstone of lung healing to prevent fibrosis. ${ }^{10}$

Several techniques of lung imaging are being elaborated with variable efficiency or side effect profiles. Computed tomography, positron emission tomography and magnetic resonance imaging have led the way to the assessment of airspace microimaging but still lack cellular/molecular resolution and specificity. ${ }^{11}$ Better performing and less invasive diagnostic methods are needed for critically ill ALI/ARDS patients, and intravital endoscopic confocal fluorescence microscopy (ECFM) is one such promising new methodology. ${ }^{12,13}$ Two minimally invasive approaches are presently studied with ECFM: (1) bronchoscopic and (2) transthoracic. A few studies have been published using both the bronchoscopic and the approaches allowing a real-time 'alveoscopy', but without testing of markers. ${ }^{12,14}$ This ECFM technology shares several advantages over conventional imaging procedures: (i) spatial and temporal resolution imaging, (ii) relatively noninvasive (safe approach, no open lung biopsy, no fixative), (iii) performed at bedside, and iv) potentially periodic follow-up.

Our hypothesis in this study was that ECFM could provide invaluable real-time insights into lung inflammation and repair processes that can affect critical care bedside physician decision-making strategy. Our objectives, by using sensitive and specific fluorescent markers, were (i) to compare images of the normal lung to ALI and (ii) to validate and compare in vivo ECFM to conventional ex vivo fluorescent and optical histopathology.

\section{MATERIALS AND METHODS Experimental Animal Models}

Pathogen-free Long-Evans rats received care in compliance with The Guide to the Care and Use of Experimental Animals from the Canadian Council of Animal Care (1993, CCAC, 2nd ed.). Protocols were approved by our institution's Ethics Committee for animal care and experimentation. ALI was induced by intratracheal (I/T) instillation of a mild dose of bleomycin $(5 \mathrm{U} / \mathrm{kg})$, or by $48-72 \mathrm{~h}$ hyperoxia exposure, as described previously. ${ }^{15}$ Neutrophil lung transit and recruitment were observed either after phorbol myristate acetate I/T instillation, hyperoxia exposure, or bleomycin I/T injection. ${ }^{15}$ At least six rats per group were examined for imaging.

\section{ECFM}

ECFM, using a dedicated research instrument (Five-1, Optiscan, Notting Hill, VIC, Australia), was performed for image acquisition. ${ }^{16}$ The Five-1 is a rigid pen-like confocal probe connected by an excitation/collection optical fiber with a $488 \mathrm{~nm}$ solid-state laser source and fluorescence detection optics sensitive from 505 to $750 \mathrm{~nm}$. When the probe-tosurface tissue contact is established, the laser power output is 'real-time' adapted by the operator-from 0 to $1000 \mu \mathrm{W}$ - to achieve appropriate contrast. The field of view is $475 \times 475 \mu \mathrm{m}$ and the axial and lateral resolutions are 7 and $0.7 \mu \mathrm{m}$, respectively. The imaging plane can be stepped down by increments of $4 \mu \mathrm{m}$, to $250 \mu \mathrm{m}$ below the surface, and resolutions vary depending on the imaging acquisition speed: $1024 \times 1024$ pixels $(0.8$ frames $/ \mathrm{s})$ or $1024 \times 512$ pixels (1.6 frames/s). ${ }^{16,17}$ Post-treatment of images was performed with Adobe Photoshop (Adobe Systems, San Jose, CA, USA). The confocal probe was introduced through either transthoracic or transdiaphragmatic routes and secured on the pleural surface.

\section{Fluorescent Markers of Lung Structure and Cellular Function}

Fluorophore-associated markers were administered topically $(\mathrm{I} / \mathrm{T}$ ) or systemically (intravenous, $\mathrm{I} / \mathrm{V}$ ) in function of their target accessibility for imaging. (1) FITC-Ricinus communis (RCA-1) and -Pisum sativum (PSA) agglutinins (Vector Laboratories, Burlingame, CA, USA); (2) FITC-labeled dextran (77 kDa, Sigma-Aldrich, St Louis, MO, USA); (3) Alexa Fluor 488-anti-RTI40 and FITC-isotypic IgG1 from murine myeloma (Sigma-Aldrich); (4) green fluorescent DNAstaining dye, YO-PRO-1 (Molecular Probes, Eugenes, OR, USA); (5) green fluorescent PKH2-labeled neutrophils (PKH2 Green Fluorescent Cell Linker Kit for Phagocytic Cell Labeling, Sigma-Aldrich); ${ }^{18,19}$ for lung cell tracking; and (6) acriflavine (Sigma-Aldrich) were the selected studied markers, as detailed in Table 1. Image acquisition with YO-PRO-1 was restricted to $10 \mathrm{~min}$ after anyway instillation in order to preserve the best signal-to noise ratio (as determined in preliminary assays).

\section{In Vivo Imaging Procedure}

The right jugular vein of experimental rats was cannulated using an angiocatheter (VWR, Mississauga, ON, Canada), and a tracheostomy was performed to secure conventional ventilation during the imaging period. ${ }^{15}$ Excess labeled I/T markers was removed by saline bronchoalveolar lavage (5-10 $\mathrm{ml})$. At the end of the experiments, lungs were sampled and frozen in Optimal Cutting Temperature (OCT, TissueTek, Sakura Finetek, Torrance, CA, USA) for ex vivo studies.

\section{Ex Vivo Lung Immunostainings}

Lung pieces were fixed $48 \mathrm{~h}$ in $10 \%$ buffered formalin, embedded in paraffin, and further stained with hematoxylin and eosin for traditional optical examination. Alternatively, lung pieces were fixed for $4 \mathrm{~h}$ in $4 \%$ paraformaldehyde at $4^{\circ} \mathrm{C}$, followed by an overnight wash in $20 \%$ cold sucrose, and then dehydrated and paraffin embedded for immunofluorescence studies. Slices of $5-\mu \mathrm{m}$ thickness were harvested and deposited on poly-L-lysine-coated slides. Rehydrated slides were incubated for $10 \mathrm{~min}$ at $37^{\circ} \mathrm{C}$ with pepsin (Digest-All; Zymed, San Francisco, CA, USA) for antigen unmasking. Nonspecific binding was prevented by incubation with $10 \%$ 
Table 1 Fluorescent marker's specifications, routes and targets for Five-1 lung imaging

\begin{tabular}{|c|c|c|c|c|}
\hline Markers & Source & $\begin{array}{c}\text { Way of } \\
\text { administration }\end{array}$ & Dosage & Imaging targets \\
\hline \multirow[t]{2}{*}{ FITC-Ricinus communis agglutinin-1 (RCA-1) } & Vector Laboratories & $1 / T$ & $0.15 \mathrm{mg} / \mathrm{ml}$ in $5 \mathrm{ml}$ saline & Type I and II epithelial cells \\
\hline & & & & Alveolar macrophages \\
\hline \multirow[t]{2}{*}{ FITC-Pisum sativum agglutinin (PSA) } & Vector Laboratories & $1 / T$ & $0.15 \mathrm{mg} / \mathrm{ml}$ in $5 \mathrm{ml}$ saline & Some type I epithelial cells \\
\hline & & & & Alveolar macrophages \\
\hline FITC-labeled dextran $77 \mathrm{kDa}$ & Sigma-Aldrich & IN & $100-500 \mu \mathrm{l}$ of $10 \mathrm{mg} / \mathrm{ml}$ saline & Lung edema and microcirculation \\
\hline Alexa Fluor 488-anti-RTI40 & Dr Dobbs Laboratory & $1 / T$ & $200 \mu \mathrm{l}$ of $100 \mu \mathrm{g} / \mathrm{ml}$ & Type I epithelial cells \\
\hline FITC isotypic $\lg G 1$ from murine myeloma & Sigma-Aldrich & $1 / T$ & $200 \mu \mathrm{l}$ of $100 \mu \mathrm{g} / \mathrm{ml}$ & None (control Abs) \\
\hline \multirow[t]{2}{*}{ YO-PRO-1 } & Molecular Probes & $\mathrm{I} / \mathrm{V}$ or $\mathrm{I} / \mathrm{T}$ & $1 \mathrm{ml}$ of $50 \mu \mathrm{M}(\mathrm{I} / \mathrm{V})$ & Apoptotic endothelial or epithelial \\
\hline & & & $5 \mathrm{ml}$ of $10 \mu \mathrm{M}(\mathrm{I} / \mathrm{T})$ & cells \\
\hline PKH2 Green Fluorescent Cell Linker Kit for & Sigma-Aldrich & I/N & $50 \times 10^{6}$ cells in $1 \mathrm{ml}$ & Neutrophils \\
\hline \multicolumn{5}{|l|}{ Phagocytic Cell Labeling } \\
\hline \multirow[t]{2}{*}{ Acriflavine } & Sigma-Aldrich & $\mathrm{I} / \mathrm{V}$ or $\mathrm{I} / \mathrm{T}$ & $500 \mu \mathrm{l}$ of $0.05 \%(\mathrm{I} / \mathrm{V})$ & Nucleus of endothelial or epithelial \\
\hline & & & $5 \mathrm{ml}$ of $0.0025-0.05 \%(\mathrm{l} / \mathrm{T})$ & cells \\
\hline
\end{tabular}

donkey serum (Zymed) and slices were recovered either with FITC-Ricinus communis, -Pisum sativum agglutinins $(0.015 \mu \mathrm{g} / \mathrm{ml}$; Vector Laboratories), or monoclonal mouse anti-RTI40 (1:1000) zenon Alexa Fluor 488 conjugated (Molecular Probes). Slides were mounted with Vectashield hardset mounting medium containing DAPI (Vector Laboratories) for nuclear counterstaining. DNA fragmentation was identified by TUNEL using the In situ Cell Death Detection Fluorescein Kit (Roche Diagnostics, Mannheim, Germany). ${ }^{20}$ Ex vivo observation of fluorescent neutrophil transit across the lung (after ECFM) was performed on OCT frozen samples and analyzed using an Axioskop 2 fluorescence microscope (Carl Zeiss, Thornwood, NY, USA).

\section{Quantification}

Thirty acquisitions from each group (normal, bleomycin, and hyperoxia rats, $n=6$ group) were randomly selected in apoptosis and neutrophil experiments for quantification purposes. Acquisitions were converted to 8-bit images and analyzed using ImageJ $1.42 \mathrm{q}$ program (National Institute of Health; http://rsb.info.nih.gov/ij). Images were pixel inverted and the background was threshold adjusted. Cells counts were performed using a particle analyzer with a size ranging from 70 to infinity.

\section{Statistical Analysis}

Results are expressed as mean \pm s.d. A Student's unpaired $t$-test analysis was selected for two-group comparisons (ie, control $v s$ bleomycin lungs) and a one-way analysis of variance (with Bartlett's test for equal variance) was used to evaluate three-group differences (ie, control $v s$ hyperoxia $v s$ bleomycin lungs). $P<0.05$ was considered as the threshold for significance.

\section{RESULTS}

Several lung areas were screened, depending on the injected marker, to obtain the best imaging quality during each experiment. Depth of focal plane, sensitivity, and laser power and fluorescence intensity-especially with combinations of markers-were adjusted to obtain optimal image contrast.

Figure 1 shows lung epithelial cell membrane labeling (type I cells) with Ricinus communis agglutinin (RCA-1) likely binding to galactose residues. Pisum sativum (PSA) lectin labeling demonstrated distinctive patterns of cell membrane staining having carbohydrate affinity for mannose moieties (data not shown). Differences in lung structure or architecture are highlighted by comparing normal lung (Figures 1a and c) with lungs that had been treated with bleomycin 3 weeks earlier, with increased alveolar, interstitial and airspace-lining cellularities, and overall enhanced parenchymal thickness (Figures $1 \mathrm{~b}$ and $\mathrm{d}$ ). Occasional strong RCA-1 labeling was observed on large cells in and along airspaces suggesting binding by macrophages and/or metaplastic (shedding) epithelial cells (Figure 1b). ${ }^{21}$ For anti-lectins of both agglutinins, specificity of in vivo stainings was further reascertained by the abolition of lung labeling with appropriate sugar preabsorption (data not shown).

Ex vivo immunofluorescence labeling with the same primary targets (ie, agglutinins, Figures $1 \mathrm{c}$ and d) was performed for ultimate comparison and validation to the imaging obtained with the Five-1 confocal endoscope.

Figure 2 represents another epithelial cell-associated protein labeling for type I cell membrane. Rat type I cell $40-\mathrm{kDa}$ sialoglycoprotein (RTI40) staining pattern has been extensively characterized earlier. ${ }^{22,23}$ As with RCA-1 labeling, differences in lung structure/architecture are highlighted by comparing normal lung (Figures $2 \mathrm{a}$ and $\mathrm{c}$ ) with late sick 

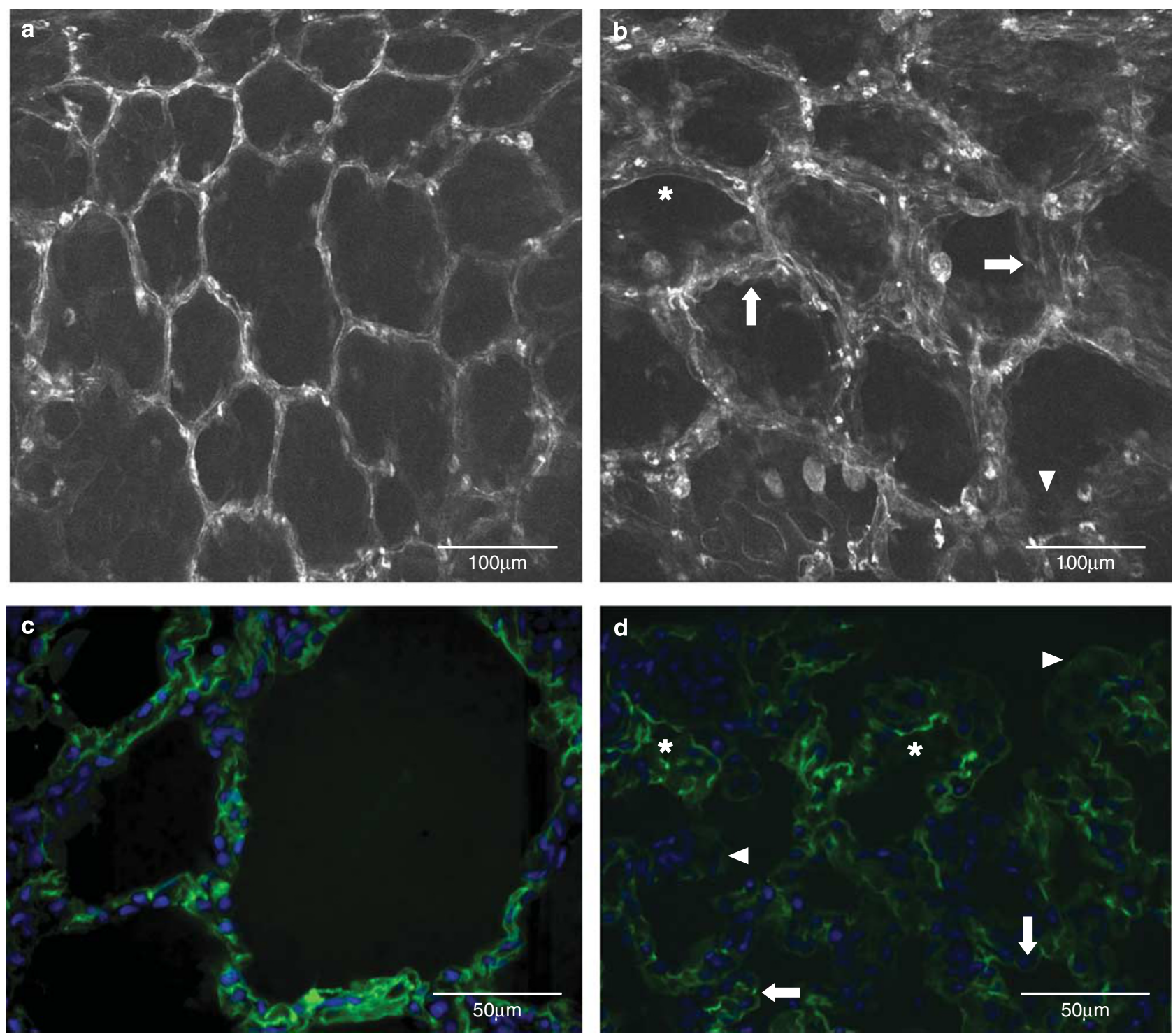

Figure 1 Lectin lung epithelial cell membrane labeling. (a) Ricinus communis agglutinin 1 lectin labeling of normal lung showing surface staining of type I epithelial cells; (b) Ricinus communis agglutinin I lectin labeling of injured lung—bleomycin model-showing enhanced parenchymal thickness together with macrophage and metaplastic epithelial cell 'big cell' staining; (c) Ex vivo labeling with Ricinus communis agglutinin 1 and DAPI of normal lung; (d) Ex vivo labeling with Ricinus communis agglutinin 1 and DAPI of bleomycin-treated lung. Examples of RCA-1 epithelial labeling in bleomycin lung are shown in panels $(\mathbf{b})$ and $(\mathbf{d}) ;{ }^{*}$, dense and continuous linear labeling; $\rightarrow$, irregular and discontinuous labeling; $\boldsymbol{}$, no labeling.

3-week-old bleomycin lung (Figures $2 \mathrm{~b}$ and $\mathrm{d}$ ), but with distinctive patterns of disorganized multilayer stratified, irregular, or discontinuous labeling (Figure 2b). Similar concentrations of nontargeted isotypic IgGs were used to validate specificity of anti-RTI40 IgG epithelial staining (Supplementary Figure E1). As the primary validation of the anti-RTI40 IgG is for ex vivo experiments, immunofluorescence labeling of processed lung samples (Figures $2 \mathrm{c}$ and d) was performed for ultimate comparison and confirmation of the imaging obtained with the Five-1 endoscope.

Figure 3 shows fluorescent neutrophil passage, recruitment, and/or diapedesis in the lung. Neutrophils were first harvested and labeled with PKH2, as described in Materials and methods, and then tracked in unchallenged or sick 2week-old bleomycin lung using the Five-1 endoscope (Figures $3 a$ and $b$ ). A few circulating neutrophils can be observed crossing the unchallenged lung microcirculation (Figures $3 \mathrm{a}$ and c), whereas numerous labeled cells can be observed within the sick lung, some of them being already translocated into airspaces (Figure 3b). Ex vivo immunofluorescence conventional studies using cryo-preserved lung samples after Five-1 screening showed similar sensitivity to ECFM (Figures $3 \mathrm{c}$ and $\mathrm{d}$ ). Indeed, semiquantitative analyses revealed 28 - and 20 -fold increases of labeled neutrophils in bleomycin lungs 

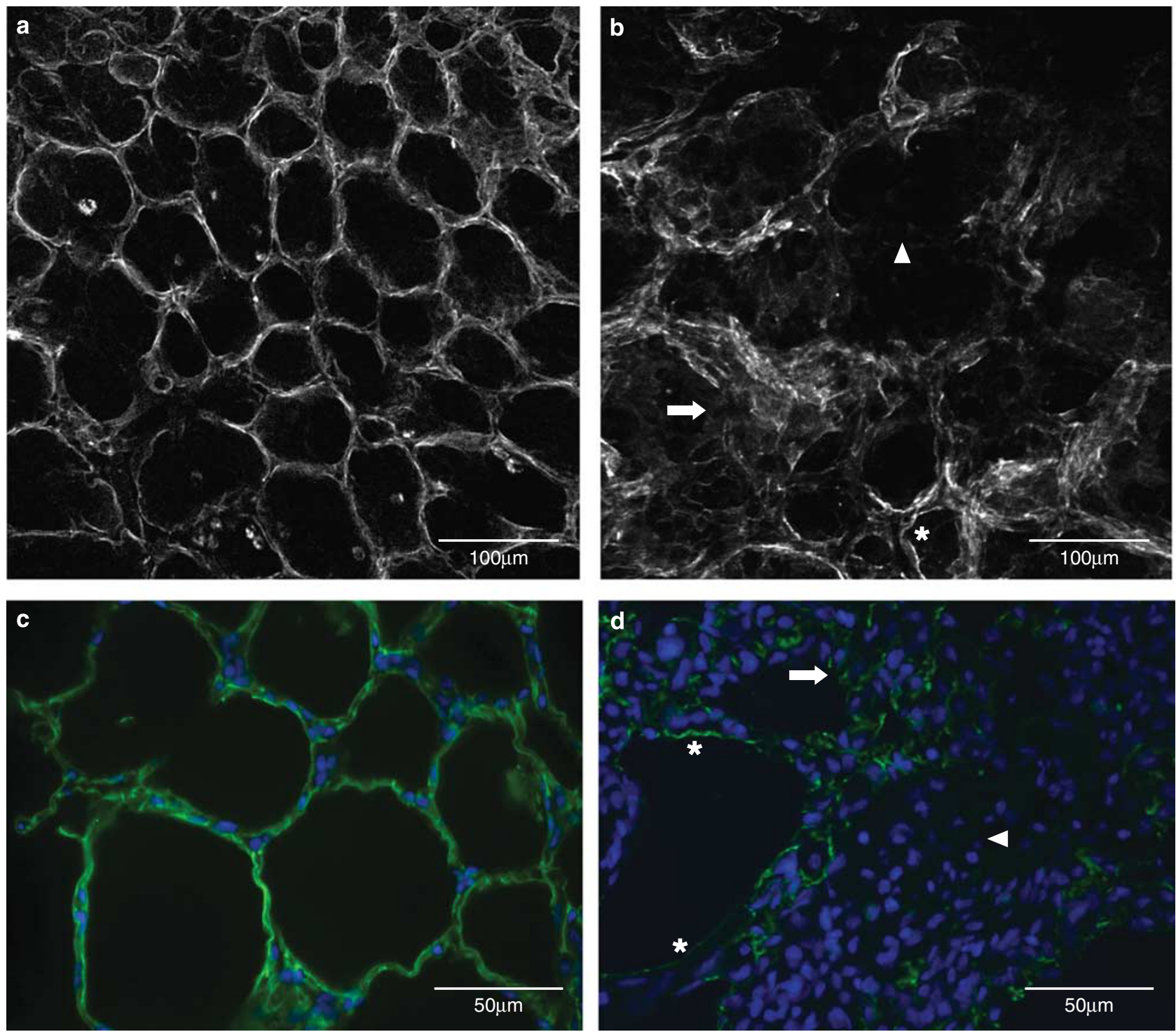

Figure 2 RTI40 lung epithelial cell membrane labeling. (a) RTI40 of normal lung showing regular, linear, and apical staining of type I epithelial cells; (b) RTI40 of injured lung — bleomycin model, at 3 weeks—showing disorganized multilayer stratified labeling; (c) ex vivo labeling with RTI40 and DAPI of normal lung type I cell membrane staining is shown, exhibiting similar pattern to (a); (d) ex vivo labeling with RTI40 and DAPI of lungs treated with bleomycin for 3 weeks. Examples of RTI40 epithelial labeling in bleomycin lung are shown in panels (b) and (d); ${ }^{*}$, dense and continuous linear labeling; $\rightarrow$, irregular and discontinuous labeling; $\gg$, no labeling.

in vivo and ex vivo $(P<0.01$ vs normal lung, respectively) (Figures $3 \mathrm{e}$ and $\mathrm{f}$ ).

Figure 4 demonstrates lung cell apoptosis using YO-PRO-1 as a DNA linker, instilled $\mathrm{I} / \mathrm{T}$ in 1 -week bleomycin $\mathrm{I} / \mathrm{T}$ challenge (Figure $4 \mathrm{~b}$ ), and in sick lungs after $60-72 \mathrm{~h}$ hyperoxia (Figure 4c), using the Five-1 endoscope. Apoptotic cells are presumably epithelial or free inflammatory cells, with possible contribution of some endothelial cells, and are numerous in contrast to occasional labeling in normal lung (Figure 4a). Ex vivo corresponding apoptotic levels using TUNEL assays (comparable method for detection of DNA commitment in cell apoptosis) detected enhanced labeled cells in bleomycin and hyperoxic lungs vs control lung (Figures $4 \mathrm{e}$ and $\mathrm{f} v s \mathrm{~d}$ ). Semiquantitative analyses revealed 25and 6.5-fold increases of labeled apoptotic cells in bleomycin lungs, as well as 9- and 6-fold corresponding increases in hyperoxic lungs in vivo and ex vivo $(P<0.001$ vs normal lung, respectively) (Figures $4 \mathrm{~g}$ and $\mathrm{h}$ ).

Figure 5 shows FITC-dextran plasma labeling in the normal lung (early phase), with interstitial microcirculation contrast and moving blood cells negatively visible (Figure 5a). A 72-h hyperoxia-exposed lung at the same phase shows early and variable diffusion of FITC-dextran outside the capillary network with interstitial and alveolar space labeling 

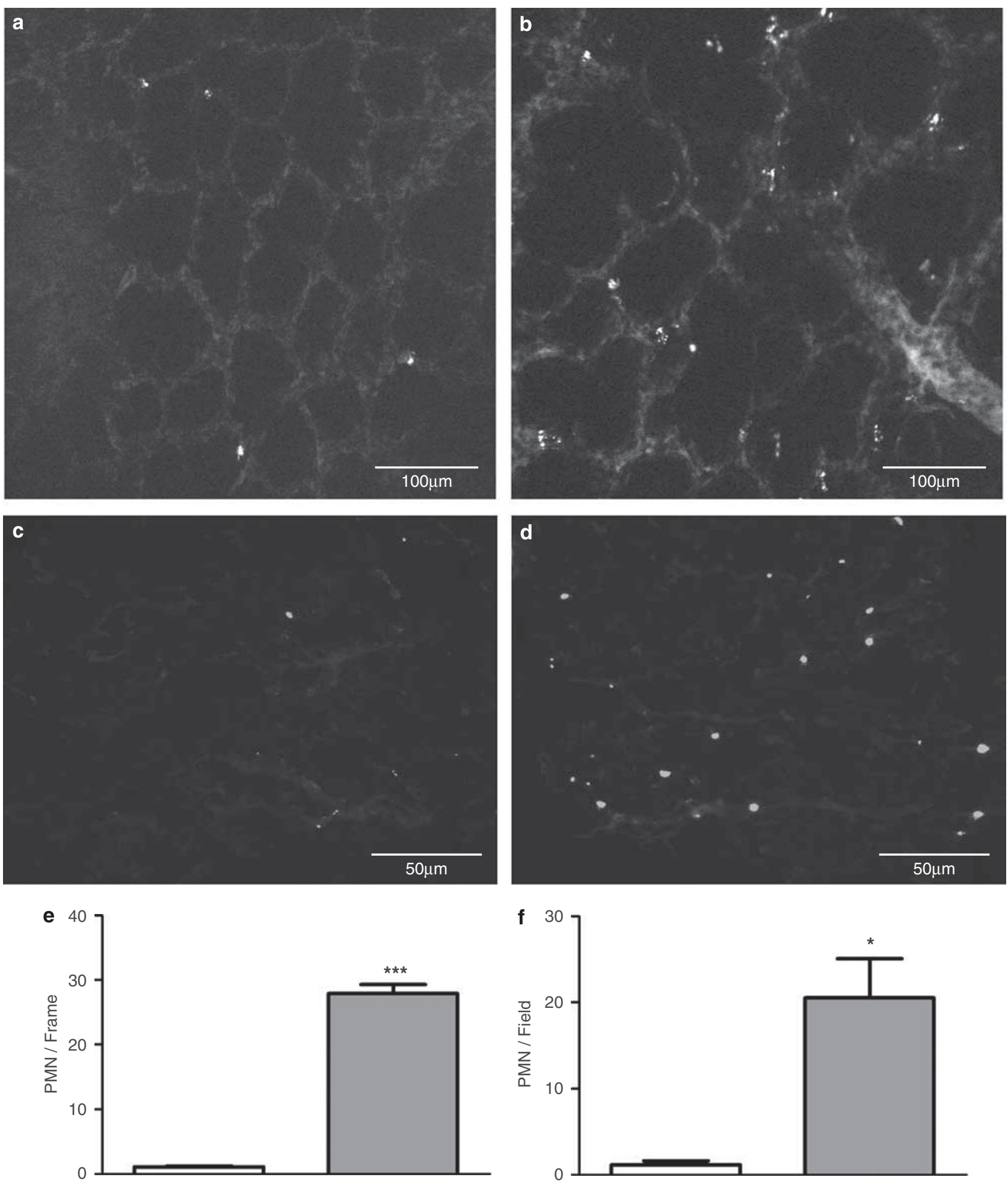

Figure 3 Lung neutrophil labeling. (a) PKH2-dyed white neutrophils in normal lung; a few circulating cells can be viewed; (b) same as (a) in injured lungsbleomycin model-showing more labeled white circulating cells including some that are translocated into airspaces; (c) ex vivo viewing of PKH2-dyed green neutrophils crossing normal lung; (d) same as (c) in lung treated with bleomycin for 2 weeks, with more labeled cells; (e) in vivo quantitation of PKH2-labeled neutrophils in the normal (white bar) and bleomycin lungs (gray bar) (mean \pm s.d., $n=6$ ); (f) ex vivo quantitation of PKH2-labeled neutrophils in the normal (white bar) and bleomycin lungs (gray bar) (mean \pm s.d., $n=6$ ). ${ }^{* *} P<0.0001$ and ${ }^{\star} P<0.01$ vs normal. 

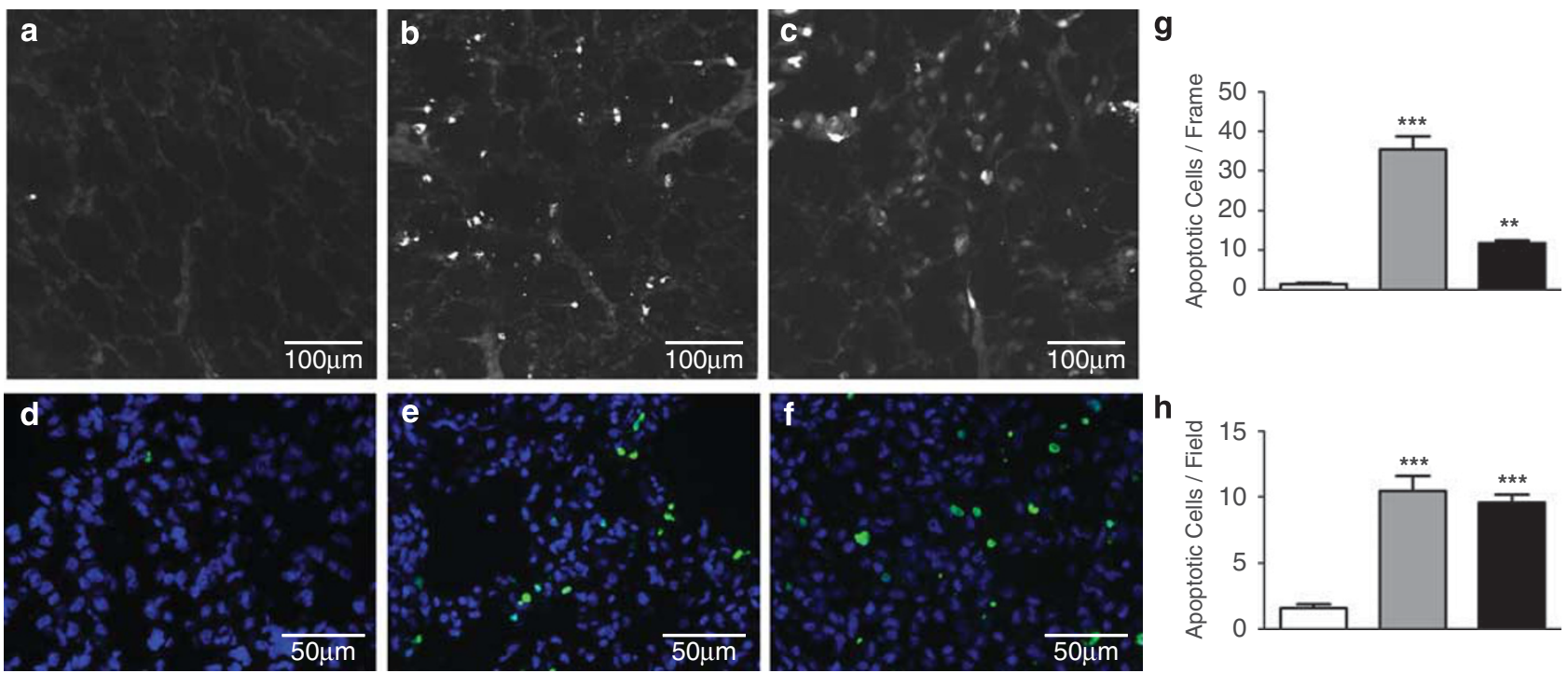

Figure 4 Visualization of lung cell apoptosis (using two different DNA end-terminal markers). YO-PRO-1 (I/T instilled) labeling with FITC-dextran (I/V) counterstaining in normal (a), bleomycin (b), and hyperoxic lungs (c). Ex vivo labeling of normal (d), bleomycin (e), and hyperoxic (f) lungs using TUNEL. (g) In vivo quantitation of apoptotic cells detected by YO-PRO-1 in the normal (white bar), hyperoxic (gray bar), and bleomycin lungs (black bar) (mean \pm s.d., $n=6)$. (h) Ex vivo quantitation of apoptotic cells detected by TUNEL in the normal (white bar), hyperoxic (gray bar), and bleomycin lungs (black bar) (mean \pm s.d., $n=6$ ). ${ }^{* * *} P<0.0001$ and ${ }^{* * P}<0.001$ vs normal.

(Figure $5 b$ ). Figures $5 c$ and $d$ show the hematoxylin and eosin corresponding pattern, in normal and hyperoxic lungs, respectively, with interstitial and alveolar edema for the latter (Figure 5d).

Though the Five- 1 system does not allow video-rate image acquisition, short sequences of consecutive image acquisition are shown, exhibiting short-time microvascular network distribution of FITC-dextran (after I/V injection) in normal (Supplementary Figure E2) and hyperoxia-exposed edema lungs (Supplementary Figure E3) (see in the online data Supplement).

\section{DISCUSSION}

In this study, lung distal airspaces from rodents were directly observed in vivo at a cellular/molecular level using a fluorescence confocal endoscope and without any tissue sampling or fixation procedures. Different pathophysiological mechanisms and structures of the lung were observed with the following objectives: (i) compare normal $v s$ sick lungs, and in vivo vs ex vivo techniques (as gold standard assessment), and (ii) address key elements committed in early ALI/ARDS and repair events (ie, epithelial cell recognition and repair processes including apoptosis, neutrophil tracking and recruitment in the lung, blood microcirculation, and lung edema). These pathophysiological processes are also intimately related to each other and critically relevant in the ARDS natural history.

The data presented herein show qualitative and semiquantitative comparisons of imaging obtained from in vivo intravital ECFM using the Five-1 instrument, and from ex vivo conventional techniques using processed samples as a gold standard method in the observation of lung microstructures. In vivo imaging, however, brings new aspects and potential for in situ repetitive assessments of epithelial cell repair processes, neutrophil recruitment, blood microcirculation networking with vessel leakage, and edema, and thus dynamic observation/monitoring after therapeutic changes-including alteration of mechanical ventilation parameters - that cannot be carried out by open lung biopsy.

\section{Epithelial Membrane Imaging: Lectin and RTI40 Labeling}

Distal airspace epithelial repair is a cornerstone and 'doubleedged-sword' event of lung repair after ALI/ARDS. On the one hand, it is critical for functional restoration, edema resolution, and fibrotic scar prevention, and on the other, it is possible that the epithelial-to-mesenchymal cell transition pathway can lead to abnormal fibrotic wounding. ${ }^{10,24-26}$ Cellselective and phenotype-specific markers for investigating injury and repair of the alveolar epithelium are thus essential to observe both anatomical and functional recovery. ${ }^{21}$ Lung structural membrane elements are useful in this way, and epithelial carbohydrate lectins with type I cell sialoglycoprotein RTI40 were appropriate markers. RCA-1 mainly recognizes lung type I epithelial cells, ${ }^{21,27}$ but also type II cell apical and basolateral plasma membranes, ${ }^{28}$ as well as other cell type though weakly (eg, monocytes or macrophages, endothelial cells). A dense and continuous linear staining of the alveolar epithelium was seen with RCA-1 by using in vivo ECFM as well as in ex vivo conventional assays, and this 

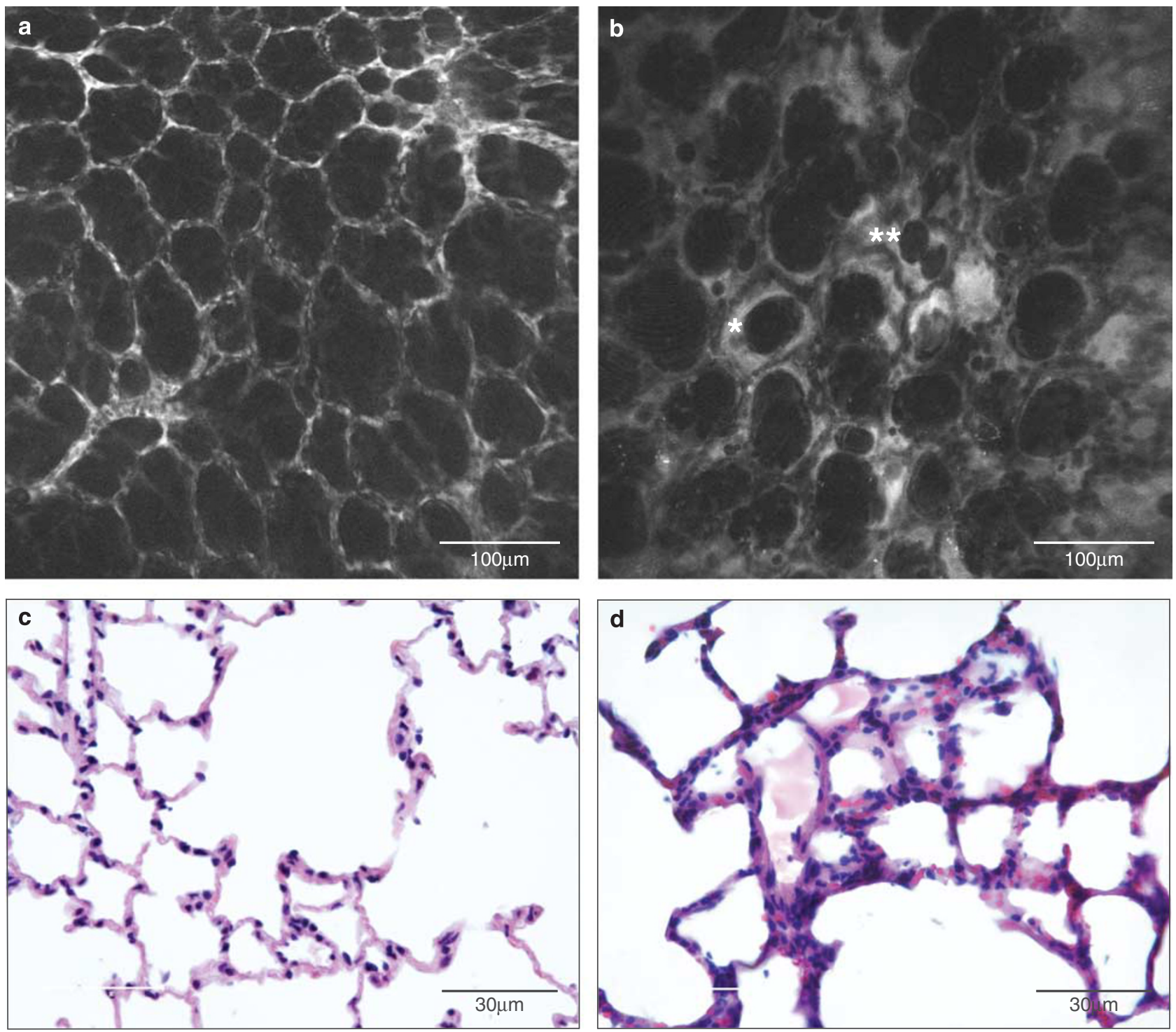

Figure 5 Visualization of lung edema and imaging was performed within 5 min after FITC-dextran intravenous injection. (a) Normal lung showing interstitial microcirculatory network and moving blood cells negatively visible. (b) A 3-day hyperoxia-exposed lung showing heterogeneous diffusion of dextran outside microvessel network leading to interstitial and alveolar space labeling. ${ }^{*}$ Shows alveolointerstitial edema (intermediate stage) and ${ }^{* *}$ shows massive, but localized, alveolar filling with stain-free microvessels. (c) Ex vivo labeling with H\&E of normal lung. (d) Ex vivo labeling with H\&E of hyperoxic lung with interstitial and alveolar edema.

pattern turned patchy, irregular, and discontinuous in the bleomycin lung after 3 weeks due to membrane blebbing; thickening of the alveolar walls and surface repair processes.

The integrity of the alveolar epithelium was also investigated using a marker for pulmonary parenchymal injury. RTI40 is a protein expressed on the apical plasma membrane of type I epithelial cells. ${ }^{22}$ The in vivo visualization of the marked antibody against RTI40 revealed parenchymal enlargement with irregular and discontinuous labeling in the bleomycin model as compared to the normal lung, whereas nonspecific labeled IgGs showed lack of staining. It also showed cellular hyperplasia, enhanced matrix deposition, and abnormal interstitial cells proliferation. In accordance with the study by Koslowski et al, ${ }^{29}$ the in vivo imaging and the ex vivo immunofluorescence showed areas of variable RTI40 protein staining at the alveolar surface (from no to irregulardiscontinuous labeling, with foci of 'normal' or already restored alveolar epithelium) 3 weeks following bleomycin administration. In this regard, taking into consideration that the dose of bleomycin I/T instilled was mild (1.5 vs $7 \mathrm{TU}$ per $\mathrm{rat}^{30}$ ) and though several brown areas were obviously visible macroscopically, IgGs were topically applied and should not normally have accessed nonatelectatic open airways and 
spaces in vivo (ie, less injured areas), whereas they will have contacted these tissues in ex vivo fixated samples.

\section{Lung Neutrophil Tracking and Recruitment}

Moving cells, such as inflammatory cells, can also be tracked by ECFM using the Five 1 instrument. Nonactivated neutrophils have a half-life of only $4-10 \mathrm{~h}$ before marginating and entering tissue pools, surviving for 1-2 days. Systemic half-life is significantly shorter in individuals suffering from infectious or acute inflammatory diseases in which requirement for newly recruited and primed neutrophils increases considerably, but tissue death is generally delayed. ${ }^{31}$ Accumulation and sequestration of activated neutrophils in microvessels is observed early on and is a hallmark of ALI/ ARDS. $^{31,32}$ Using either ex vivo neutrophil labeling-reinjection or systemic intravenous in vivo direct labeling techniques, ECFM was able to detect real-time passage of small numbers of fluorescent cells across the normal lung microcirculatory network, neutrophil sequestration diapedesis after bleomycin I/T challenge, which peaks between 2 and 17 days after instillation ${ }^{33}$, or nearly immediate neutrophil locoregional recruitment after catheter-guided phorbolmyristate acetate $\left(10^{-8} \mathrm{M}\right)$ segmental lung challenge (not shown). It is also noteworthy that ECFM could also potentially allow tracking of other inflammatory or progenitor cells.

\section{Lung Cell Apoptosis}

Lung cell apoptosis is a regulated form of death whose deregulation can lead to wound failure, fibrosis, or sustained inflammation. ${ }^{34}$ Of the numerous cells involved in ALI/ ARDS, some exhibit intense and accelerated apoptosis (eg, epithelial and endothelial cells, lymphoid cells), and others a delayed apoptosis (eg, neutrophils, mesenchymal cells). ${ }^{31-35}$ Imaging cell apoptosis in tissues of living animals has been a topic in expansion over the past few years. Several studies investigated the subject by using alternative technologies such as magnetic resonance imaging ${ }^{36,37}$ or positron emission tomography with radiolabeled annexin $\mathrm{V}^{38}$ However, the resolution of these devices is appropriate to determine region of interest but does not allow the detection of apoptosis at the cellular-scale level. In addition, most of the markers used in vivo to date showed upstream apoptotic events, whereas the fluorescent DNA-staining dye used in this study, YOPRO-1, revealed DNA fragmentation characteristic of downstream terminal apoptosis. This dyed linker is selectively for cross-permeant membranes of apoptotic cells and has already been validated for in vivo use. ${ }^{39}$ After experimental injury, I/T injection of YO-PRO-1 was particularly accurate for lung apoptosis imaging using ECFM, and abundant nuclear staining of apoptotic cells-presumably mainly alveolar epithelial cells, with few inflammatory, endothelial, and interstitial cells-was visualized in both bleomycin and hyperoxic models. ${ }^{40,41}$ Hyperoxic lungs preferentially presented a sparse or homogeneous pattern, whereas bleomycin lungs more likely showed patchy clusters of apoptotic cells. Indeed, hyperoxic lungs are recognized by injury and death of more alveolar endothelial than epithelial cells, whereas the bleomycin lung produces more alveolar epithelial cell injury and death, ${ }^{20,34,35,40,42}$ with respect to distinctive natural histories of both models. Indeed, cellular source of apoptosis and reagent accessibility may explain part of the apparent discrepancy between the relatively lower detection rate of apoptotic cells detected by the Five-1 in hyperoxic $v$ s bleomycin lungs, compared with the similar detection rates observed using TUNEL. ECFM observations correlated easily with the traditional TUNEL or ISEL techniques on ex vivo fixated lung sections. ${ }^{20,40,43}$

\section{Lung Microcirculation and Edema}

Pulmonary edema is another critical feature for monitoring parameters of ALI/ARDS by bedside ICU physicians, ${ }^{44}$ although it is often underestimated and difficult to assess. Although the epithelial barrier is less permeable than the endothelium physiologically, the loss of epithelial cells in distal airspaces of ALI/ARDS lungs is common and a cornerstone of the repair processes. ${ }^{9,10,24-26,34,35,40}$ There is growing evidence that lung permeability should be considered as a target for new therapy in ALI/ARDS: current knowledge on catecholamines-induced improved fluid clearance, ${ }^{10,24,44-46}$ trends for increase of ventilator-free days with decrease in extravascular lung water ${ }^{46}$ and evidence for reduction of ventilatory needs with conservative $v s$ liberal fluid resuscitation. ${ }^{47}$ However, validated and reliable procedures for lung permeability assessment are still lacking, although FITC-dextran ${ }^{48}$ and semiquantitative double-isotope imaging technique (using albumin and transferrin) ${ }^{49}$ have become popular experimentally, in vivo as well as ex vivo.

In this study, we presented endoscopic confocal imaging data allowing visualization of dextran 'real-time' extravasation in a model of lung hyperoxia, without quantitative purposes. Edema at this time was clinically and macroscopically obvious, however, astonishingly variable and inhomogeneous on a dynamic and microscopic basis. This emerging endomicroscopic technique is able to offer physicians an additional tool to evaluate actual edema status in localized areas, in addition to conventional methods (eg, Piccoß, wedge pressure ${ }^{44}$ ).

\section{Limitations of This Study}

This study is preliminary because of the use of a limited number of fluorescent markers to assess ALI/ARDS. In addition, these exogenous fluorescent reagents, with exception to fluoresceinated dextran, are not presently approved for human use, and in such a case, using these reagents in their present form would not be necessarily cost-effective. The Five-1 instrument is a unichannel (FITC scale) system, does not allow co- (expression) localization labeling, and its performance is comparable to other devices such as the Cellvizioß. Only qualitative or semiquantitative imaging was provided at this time. Further, extended and refined 
assessments of lung cellular and structural are mandatory with repetitive time-frame screening, for instance by using selective permeant peptides delivered in the airways. Ultimately, an algorithmic strategy for real-time diagnostic lung assessment should be validated (in small and large animals), innocuity of repetitive fluorescent labeling procedures ascertained, and first phases of human studies should be initiated.

\section{Conclusions}

This study is the first to our knowledge to report in vivo realtime endomicroscopic assessment of lung distal airspaces in normal and acutely injured lungs using specific fluorescent markers. Two recent reports using a similar device reported lung airspace imaging without labeling with specific markers but with natural autofluorescence ${ }^{12,14}$ and did not address ALI. With these preliminary data, progress on an algorithmic labeling profile of unrepairing lungs using this fluorescence microendomicroscopic method is foreseen and this could conceivably lead to a clinically useful tool for ICU physicians.

Supplementary Information accompanies the paper on the Laboratory Investigation website (http://www.laboratoryinvestigation.org)

\section{ACKNOWLEDGEMENT}

We thank Line Bilodeau for her helpful technical assistance. This work was supported by the Reseau en Sante Respiratoire (RSR) du FRSQ, the CRCCHUS (PAFI 2007), a studentship grant from the Faculte de Genie de I'UDS $(\mathrm{CF})$, and Optiscan, Victoria, Australia, which supplied the Five-1 device.

\section{DISCLOSURE/CONFLICT OF INTEREST}

The authors declare no conflict of interest.

1. Bernard GR, Artigas $\mathrm{A}$, Brigham $\mathrm{KL}$, et al. The American-European Consensus Conference on ARDS. Definitions, mechanisms, relevant outcomes, and clinical trial coordination. Am J Respir Crit Care Med 1994;149:818-824.

2. McIntyre Jr RC, Pulido EJ, Bensard DD, et al. Thirty years of clinical trials in acute respiratory distress syndrome. Crit Care Med 2000;28:3314-3331.

3. Rubenfeld GD, Caldwell E, Peabody E, et al. Incidence and outcomes of acute lung injury. N Engl J Med 2005;353:1685-1693.

4. ARDSNetwork. Ventilation with lower tidal volumes as compared with traditional tidal volumes for acute lung injury and the acute respiratory distress syndrome. The acute respiratory distress syndrome network. N Engl J Med 2000;342:1301-1308.

5. Wheeler AP, Bernard GR. Acute lung injury and the acute respiratory distress syndrome: a clinical review. Lancet 2007;369:1553-1564.

6. Donati SY, Papazian L. Role of open-lung biopsy in acute respiratory distress syndrome. Curr Opin Crit Care 2008;14:75-79.

7. Kao KC, Tsai YH, Wu YK, et al. Open lung biopsy in early-stage acute respiratory distress syndrome. Crit Care 2006;10:R106.

8. Schwarz Ml, Albert RK. 'Imitators' of the ARDS: implications for diagnosis and treatment. Chest 2004;125:1530-1535.

9. Tomashefski Jr JF. Pulmonary pathology of the adult respiratory distress syndrome. Clin Chest Med 1990;11:593-619.

10. Berthiaume $Y$, Lesur $O$, Dagenais A. Treatment of adult respiratory distress syndrome: plea for rescue therapy of the alveolar epithelium. Thorax 1999;54:150-160.

11. Brown RH, Irvin CG, Allen III GB, et al. An official ATS conference prodeedings: advances in small-animal imaging application to lung pathophysiology. Proc Am Thorac Soc 2008;5:591-600.
12. Thiberville $\mathrm{L}$, Moreno-Swirc $\mathrm{S}$, Vercauteren $\mathrm{T}$, et al. In vivo imaging of the bronchial wall microstucture using fibered confocal fluorescence microscopy. Am J Respir Crit Care Med 2007;175:22-31.

13. Flusberg BA, Cocker ED, Piyawattanametha W, et al. Fiber-optic fluorescence imaging. Nature Methods 2005;2:941-950.

14. Thiberville $L$, Salaün $M$, Lachkar $S$, et al. Human in-vivo fluorescence microimaging of the alveolar ducts and sacs during bronchoscopy. Eur Respir J 2009;33:974-985.

15. Lesur O, Hermans C, Chalifour JF, et al. Pneumoprotein (CC-16) vascular transfer during mechanical ventilation in rats: effect of KGF pretreatment. Am J Physiol Lung Cell Mol Physiol 2003;284:L410-L419.

16. Fournier C, Moleski L, Charrette PG, et al. Towards in vivo imaging of lung repair: current issues and future directions. Journal of Organ Dysfunction 2008; , special issue (ISRD 2008 Shangaï); 71-78.

17. Goetz M, Fottner C, Schirrmacher E, et al. In-vivo confocal real-time mini-microscopy in animal models of human inflammatory and neoplastic diseases. Endoscopy 2007;39:350-356.

18. Albertine $\mathrm{KH}$, Gee $\mathrm{MH}$. In vivo labeling of neutrophils using a fluorescent cell linker. J Leukoc Biol 1996;59:631-638.

19. Yuan Y, Fleming BP. A method for isolation and fluorescent labeling of rat neutrophils for intravital microvascular studies. Microvasc Res 1990;40:218-229.

20. Lesur O, Brisebois M, Thibodeau A, et al. Role of IFN-gamma and IL-2 in rat lung epithelial cell migration and apoptosis after oxidant injury. Am J Physiol Lung Cell Mol Physiol 2004;286:L4-L14.

21. McElroy MC, Kasper M. The use of alveolar epithelial type I cellselective markers to investigate lung injury and repair. Eur Resp J 2004:24:664-673.

22. McElroy MC, Pittet JF, Hashimoto S, et al. A type I cell-specific protein is a biochemical marker of epithelial injury in a rat model of pneumonia. Am J Physiol 1995;268:L181-L186.

23. Dobbs LG, Williams MC, Gonzalez R. Monoclonal antibodies specific to apical surfaces of rat alveolar type I cells bind to surfaces of cultured, but not freshly isolated, type II cells. Biochim Biophys Acta 1988;970:146-156.

24. Matthay MA, Robriquet L, Fang X. Alveolar epithelium: role in lung fluid balance and acute lung injury. Proc Am Thorac Soc 2005;2:206-213.

25. Gropper MA, Wiener-Kronish J. The epithelium in acute lung injury/ acute respiratory distress syndrome. Curr Opinion in Crit Care 2008;14:11-15.

26. Selman M, Pardo A. Role of epithelial cells in idiopathic pulmonary fibrosis. Proc Am Thorac Soc 2006;3:364-372.

27. Brandt AE. Cell surface saccharides of rat lung alveolar type I and type Il cells. Fed Proc 1982;41:755-761.

28. Taatjes DJ, Barcomb LA, Leslie KO, et al. Lectin binding patterns to terminal sugars of rat lung alveolar epithelial cells. J Histochem Cytochem 1990;38:233-244.

29. Koslowski R, Dobbs LG, Wenzel KW, et al. Loss of immunoreactivity for RTI40, a type I cell-specific protein in the alveolar epithelium of rat lungs with bleomycin-induced fibrosis. Eur Respir J 1998;12: 1397-1403.

30. Bowden DH. Experimental induction of pulmonary fibrosis. In: Gil J (ed). Models of Lung Disease. Microscopy and Structural Methods, vol 47. Lung Biology in Health and Disease, Marcel Dekker Inc: New-York and Basel, 1990, chapt 22: pp 735-752.

31. Abraham E. Neutrophils and acute lung injury. Crit Care Med 2003;31:S195-S199.

32. Lee WL, Downey GP. Neutrophil activation and acute lung injury. Curr Opin Crit Care 2001;7:1-7.

33. Sato N, Suzuki Y, Nishio K, et al. Role of ICAM-1 for abnormal leukocyte recruitment in the microcirculation of Bleomycin-induced fibrotic lung injury. Am J Respir Crit Care Med 2000;161:1681-1688.

34. Martin TR, Hagimoto N, Nakamura M, et al. Apoptosis and epithelial injury in the lungs. Proc Am Thorac Soc 2005;2:214-220.

35. Uhal BD. The role of apoptosis in pulmonary fibrosis. Eur Respir Rev 2008;17:138-144.

36. Zhao $M$, Beauregard DA, Loizou $L$, et al. Non-invasive detection of apoptosis using magnetic resonance imaging and a targeted contrast agent. Nature Med 2001;7:1241-1244. 
37. Hakumaaki JM, Brindle KM. Techniques: visualizing apoptosis using nuclear magnetic resonance. Trends Pharmacol Sci 2003;24: 146-149.

38. Blankenberg FG, Katsikis PD, Tait JF, et al. In vivo detection and imaging of phosphatidylserine expression during programmed cell death. Proc Natl Acad Sci 1998;95:6349-6354.

39. Al-Gubory $\mathrm{KH}$. Fibered confocal fluorescence microscopy for imaging apoptotic DNA fragmentation at the single-cell level in vivo. Exp Cell Res 2005;310:474-481.

40. Li X, Rayford H, Uhal BD. Essential roles of angiotensin receptor AT1a in bleomycin-induced apoptosis and lung fibrosis in mice. Am J Pathol 2003;163:2523-2530.

41. Barazzone $C$, White $C$. Mechanisms of cell injury and death in hyperoxia. Am J Respir Cell Mol Biol 2000;22:517-519.

42. Crapo JD, Barry BE, Foscue HA, et al. Structural and biochemical changes in rat lungs occurring during exposures to lethal and adaptive doses of oxygen. Am Rev Respir Dis 1980;122:123-143.

43. Howlett CE, Hutchison JS, Veinot JP, et al. Inhaled nitric oxide protects against hyperoxia-induced apoptosis in rat lungs. Am J Physiol Lung Cell Mol Physiol 1999;277:L596-L605.
44. Isakow W, Schuster DP. Extravascular lung water measurements and hemodynamic monitoring in the critically ill: bedside alternatives to the pulmonary artery catheter. Am J Physiol Lung Cell Mol Physiol 2006;291:L1118-L1131.

45. Perkins GD, McAuley DF, Thickett DR, et al. The $\beta$-agonist lung injury trial (BALTI). A randomized placebo-controlled clinical trial. Am J Respir Crit Care Med 2006;173:281-287.

46. Martin GS, Eaton S, Mealer M, et al. Extravascular lung water in patients with severe sepsis: a prospective cohort study. Crit Care 2005;9:R74-R82.

47. The National Heart, Lung, and Blood Institute Acute Respiratory Distress Syndrome (ARDS) Clinical Trials Network. Comparison of two fluid-management strategies in acute lung injury. $\mathrm{N}$ Engl J Med 2006;354:2564-2575.

48. Guery BP, Nelson S, Viget N, et al. Fluorescein-labeled dextran concentration is increased in BAL fluid after ANTU-induced edema in rats. J Appl Physiol 1998;85:842-848.

49. DeProst N, Dreyfuss D, Saumon G. Evaluation of two-way protein fluxes across the alveolocapillary membrane by scintigraphy in rats. J Appl Physiol 2007;102:794-802. 